\title{
Local Intestinal ACE-Like Activity and Corticosterone Production in Hypertensive and Aging Rats
}

\author{
Hanne Salmenkari*, Mervi Holappa, Aino Siltari, Riitta Korpela, Heikki Vapaatalo
}

Medical Faculty, Pharmacology, University of Helsinki, Helsinki, Finland

Email: *hanne.salmenkari@helsinki.fi

How to cite this paper: Salmenkari, $\mathrm{H}$., Holappa, M., Siltari, A., Korpela, R. and Vapaatalo, H. (2018) Local Intestinal ACE-Like Activity and Corticosterone Production in Hypertensive and Aging Rats. Pharmacology \& Pharmacy, 9, 27-37.

https://doi.org/10.4236/pp.2018.91003

Received: December 8, 2017

Accepted: January 16, 2018

Published: January 19, 2018

Copyright () 2018 by authors and Scientific Research Publishing Inc. This work is licensed under the Creative Commons Attribution International License (CC BY 4.0).

http://creativecommons.org/licenses/by/4.0/

\begin{abstract}
The renin angiotensin system (RAS) participates in inflammatory processes, as either a pro-inflammatory or an anti-inflammatory mediator. Components of RAS, such as angiotensin-converting enzyme (ACE), have been detected locally in the gut epithelium. In addition, the anti-inflammatory steroid, corticosterone, is produced in the gut. Hypertension and aging evoke a low-grade inflammatory process in the vascular endothelium. It is not known whether they induce a similar low-grade inflammation in the intestine and if the low-grade inflammation would evoke an activation of ACE and an elevation of corticosterone production. These two variables were measured in ileum and colon of 9- and 26-week old spontaneously hypertensive rats (SHR) and their normotensive Wistar-Kyoto (WK) controls. ACE-activity, measured via the formation of histidyl-leucine from hippuryl-histidyl-leucine in the tissue homogenate samples, was similar in the ileum and colon of young animals although the ileum of the young normotensive animals displayed the lowest level. In the old animals, the ACE activity was higher in the ileum than in the colon, especially in normotensive rats. Corticosterone production was measured as corticosterone concentration in the supernatants of ileum or colon after a 90-min ex vivo incubation. Corticosterone production was higher in ileum than in colon in both SHR and WK. No clear evidence was seen for age-dependency or for an effect of hypertension in the measured variables in the intestine. Thus, the putative low-grade inflammation in the intestine in aging or hypertension is not a strong enough stimulus to elevate corticosterone production or activate ACE.
\end{abstract}

\section{Keywords}

Intestine, ACE, Corticosterone, Hypertension, Aging 


\section{Introduction}

The components of the renin angiotensin system (RAS) in circulation have a crucial role in the regulation of blood pressure. From a therapeutic point of view, the angiotensin-converting enzyme (ACE), the enzyme forming active angiotensin II from the much less vasoconstrictive peptide, angiotensin I, is the target for antihypertensive ACE-inhibitors. In addition to circulating RAS, research has revealed the presence of a local RAS in vasculature. Furthermore, components of RAS have been detected in many other tissues/organs (see, Gonzalez-Villalobos et al., 2013) [1]. Already more than 25 years ago, different peptidases, including ACE (also known as kininase II) were detected in the brush border of human and swine intestine [2] as well as in rat intestine and vascular surface membranes [3]. Later, rat intestinal ACE was purified; its expression, activity and properties were revealed from a total cell membrane fraction of rat intestinal mucosa [4]. The classical RAS and ACE promote the production of the potent vasoconstrictor peptide, angiotensin II, which stimulates adrenal aldosterone synthesis to increase water and sodium reabsorption and potassium excretion in the kidney and intestine. The classical RAS is considered as pro-inflammatory i.e. the overexpression of the angiotensin I - (ACE) - angiotensin II - angiotensin I receptor type 1 - axis in the gut as it exacerbates inflammation [5]. There are several reports where either RAS-inhibition (e.g. ACE-inhibitors and angiotensin receptor type 1 blockers) or knockout of RAS components has alleviated experimental colitis [6]-[17]. We have recently shown that ACE protein is released from the intestine following inflammation and that ACE-inhibitors reduce the amounts of Ace and Angiotensinogen mRNA in inflamed colon [18]. The key enzyme of the alternative RAS, ACE2, stimulates the formation of Ang (1-7), which rather selectively stimulates vasodilatory Mas receptors, and opposes many actions of the classical RAS pathway and alleviates inflammation [7] [19].

Several studies have shown that glucocorticoids are synthetized in extra-adrenal tissues e.g. the gastrointestinal epithelium [20] [21] [22] [23] [24]. Local synthesis of corticosterone has been detected in various extra-adrenal tissues including lymphoid organs, intestine, skin, brain and possibly in heart [20]. The evidence for local synthesis includes the presence of steroidogenic enzymes and high levels of steroids even in adrenalectomized animals. Glucocorticoids synthetized in the adrenal cortex, under the control of ACTH, are important stress hormones with potent physiological and therapeutic anti-inflammatory properties. In the intestine, inflammation increases corticosterone production to balance local immune responses [22] [25].

Aging causes a decline in many gut-related functions, starting from less sense of taste in the mouth down to decreased motility (obstipation) of the colon. Aging is associated with structural and functional mucosal defects, diminished immune defence, increased inflammation due to oxidative stress, and dysfunction of the epithelial barrier [26] [27]. Low-grade, chronic, systemic inflammation in aging 
without infection e.g. in the colon, has been named "inflammaging" [28]. The aging intestine produces endogenous pro-inflammatory molecules which together with gut microbiota evoke a state of chronic inflammation in the elderly [29].

Our hypothesis is that different forms of low grade inflammation are present in the gut epithelium, in a similar manner to that in the vascular endothelium of hypertensive and/or aging individuals. The aim of this study was to examine whether a similar phenomenon can be detected in intestine and whether it reflects or acts in parallel with the production of anti-inflammatory corticosterone.

\section{Materials and Methods}

\subsection{Animals}

We used 9 and 26-week old Spontaneously hypertensive (SHR) male rats and their normotensive controls, Wistar-Kyoto (WK) rats (Charles River Laboratories) of same ages ( $n=6$ in each group) which were housed two animals in a cage in a pathogen-free animal laboratory with a $12 \mathrm{~h}$ light/dark cycle at room temperature, and relative humidity of $55 \%$ as described previously [29]. The animals had free access to rat laboratory feed (Harlan, Rossdorf, Germany) and tap water. The protocol was included and approved by the National Animal Experimentation Committee of Finland according to EC Directive 86/609 EEC and the Finnish Experimental Animal Act 62/2006s.

\subsection{Sample Collection}

The animals were anesthetized and sacrificed with $\mathrm{CO}_{2}$ and cardiac puncture. Samples of ileum and proximal colon were excised, cleaned from adjacent fat and connective tissue, opened and cleaned from feces and rinsed with saline. In the ACE-activity assay, pieces of ileum and colon were flash frozen in liquid nitrogen and stored at $-80^{\circ} \mathrm{C}$ until homogenization. In the corticosterone production assay, approximately $1 \mathrm{~cm}$ long pieces of ileum and proximal colon were incubated in pre-oxygenated Krebs buffer $(119 \mathrm{mmol} / \mathrm{L} \mathrm{NaCl}, 25 \mathrm{mmol} / \mathrm{L} \mathrm{Na}$ $\mathrm{HCO}_{3}, 15 \mathrm{mmol} / \mathrm{L} \mathrm{KCl}, 11 \mathrm{mmol} / \mathrm{L}$ glucose, $1.6 \mathrm{mmol} / \mathrm{L} \mathrm{CaCl}{ }_{2}, 1.2 \mathrm{mmol} / \mathrm{L}$ $\mathrm{KH}_{2} \mathrm{PO}_{4}, 1.2 \mathrm{mmol} / \mathrm{L} \mathrm{MgSO}_{4}$ ) at $37^{\circ} \mathrm{C}$ for $90 \mathrm{~min}$ with gentle agitation. The chemicals were purchased from Sigma-Aldrich (St Louis, MO, USA). The samples were centrifuged at $13000 \mathrm{~g}$ for $3 \mathrm{~min}$ and the incubation supernatants were stored at $-20^{\circ} \mathrm{C}$ until assayed.

\subsection{ACE-Activity Assay}

Tissue pieces were homogenized in a cryo-bead homogenizer in $100 \mathrm{mM}$ Tris $120 \mathrm{mM} \mathrm{NaCl}, \mathrm{pH}$ 8.3. ACE activity was measured spectrophotometrically with a modified fluorometric assay with hippuryl-histidyl-leucine (HHL) as the substrate as originally described in [30]. Briefly, samples were incubated in 1:1 volume of $6 \mathrm{mM} \mathrm{HHL}-100 \mathrm{mM}$ Tris $-120 \mathrm{mM} \mathrm{NaCl}$, pH 8.3 for $60 \mathrm{~min}$ at $37^{\circ} \mathrm{C}$, after which the reaction was stopped with $0.3 \mathrm{M} \mathrm{NaOH}$ solution. 2:25 volumes of 
$20 \mathrm{mg} / \mathrm{ml} o$-phthaldialdehyde was added in the reactions followed by a 10-minute incubation at RT in dark. Then, 1:6 volumes of $3 \mathrm{M} \mathrm{HCl}$ were added and the fluorescence was measured at $355 \mathrm{~nm}$ ex, $535 \mathrm{~nm}$ em. The fluorescent intensity was plotted against an HL standard curve and ACE activity was calculated as the formation of histidyl-leucine (HL) $\mathrm{nM} / \mathrm{min}$.

Corticosterone secretion into the incubation fluid was measured by EIA kit (\#500655 Cayman Chemical, Michigan, MI, USA). The protein content of the respective tissue lysates was measured by Pierce ${ }^{\mathrm{TM}}$ BCA Protein Assay kit (Thermo Fisher Scientific, Waltham, MA, USA). The ACE activity and the amounts of corticosterone secreted into the incubation supernatant were calculated per mg total protein.

\subsection{Statistical Analyses}

Statistical analysis in the comparison of differences (Mean \pm SEM) between ileum and colon, young and old, normotensive and hypertensive animals and ileum, respectively was done with one-way ANOVA followed by Tukey's HSD post hoc test Correlations were estimated with Pearson's correlation coefficient. All statistical analyses were calculated with SPSS version 23. P-values less than 0.05 were considered statistically significant.

\section{Results}

\section{Aging modulates ACE activity differentially in WK and SHR rats}

ACE activity in ileum-tissue lysates in the two age groups (young, old) of normotensive (WK) and spontaneously hypertensive (SHR) rat are presented in Figure 1(a). The ACE activity in ileum increased with age both in WK $3.27 \pm$ $0.06 \mathrm{U} / \mathrm{mg}$ in old vs $0.73 \pm 0.03 \mathrm{U} / \mathrm{mg}$ in young) and SHR (2.23 \pm 0.06 vs $1.53 \pm$ $0.10 \mathrm{U} / \mathrm{mg}$, respectively) animals. In young animals, the ACE activity was higher in SHR rats compared to WK rats. In old animals, the situation was reversed i.e. ACE activity was higher in WK rats.

The ACE activities in the colon samples of the four different groups are summarized in Figure 1(b). Generally, ACE activity was roughly at the same level as in the ileum. Similar to the levels in ileum, ACE activity increased with age in WK rats $(2.13 \pm 0.11$ in old vs $1.29 \pm 0.08 \mathrm{U} / \mathrm{mg}$ in young) but the levels were similar in young and old SHR rats $(1.56 \pm 0.08$ vs $1.52 \pm 0.06 \mathrm{U} / \mathrm{mg}$, respectively). In line with the results in ileum, ACE activity was slightly lower in old SHR than WK rats. In young rats, there were no differences between strains in ACE activity.

\section{Corticosterone production is not dependent on age or blood pressure}

To investigate whether corticosterone production was modulated by either age or blood pressure, we measured the corticosterone secretion from ileum and colon samples into the incubation supernatant (Figure 2). Corticosterone production was approximately at the same level in both ileum and colon and neither age nor blood pressure modulated the production of corticosterone in ileum 


\section{ACE Activity}

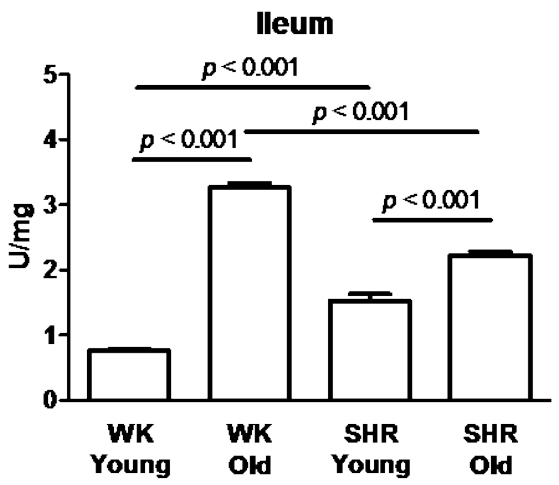

(a)
Colon

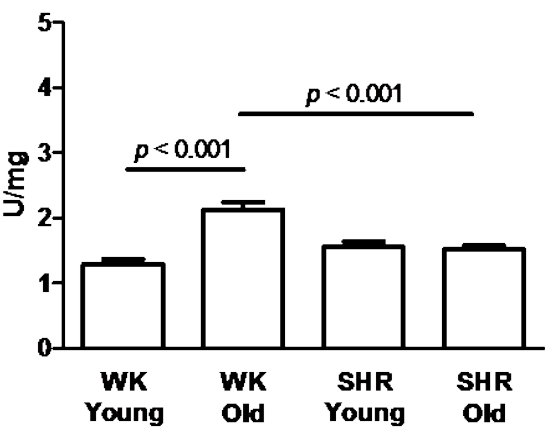

(b)

Figure 1. ACE activity in the ileum (left) and colon (right) homogenates of young and old WK and SHR rats. The values are presented as mean \pm SEM ( $n=5-6$ in each group).

\section{Corticosterone}

lleum

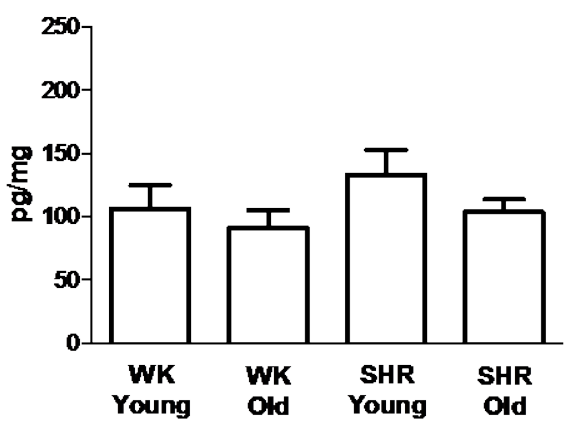

(a)

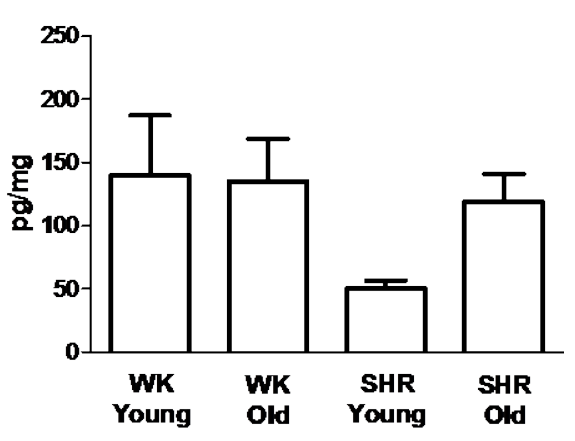

(b)

Figure 2. Corticosterone secretion in the ileum (left) and colon (right) of young and old WK and SHR rats. Corticosterone was measured in incubation supernatants after a 90-min ex vivo incubation. The values are presented as mean \pm SEM ( $n=5-6$ in each group).

(Figure 2(a)) or colon (Figure 2(b)). However, the absolute values were low in young SHR colon samples, but the difference was not statistically significant ( $p=$ 0.701 between young and old SHR).

We also tested if there was any correlation between ACE-activity in the intestinal lysates and the amount of corticosterone secreted into the incubation supernatant, but we did not find any significant correlation when all the values (young and old, WKs and SHRs) were taken into account (Figure 3). This was also the case, when the calculation was conducted separately using the ileal or colonic values (data not shown).

\section{Discussion}

Our hypothesis was that, as in the arterial endothelium [29], low-grade inflammation would be detectable with different biomarkers in intestinal epithelium. Low-grade inflammation has been reported in the vasculature of hypertensive 
animals and humans [31] [32] and as endothelial dysfunction in old animals and elderly people [33]. As shown by us [18] [23] and others [2] [3] [22] in incubations with ileal and colonic slices, ACE protein and corticosterone are released into the incubation buffer and in mice, their releases correlate with each other. In the present study, we used two strains of rats, spontaneously hypertensive (SHR) and their normotensive controls, Wistar-Kyoto (WK) rats. Additionally, we studied two different age groups, young ( 9 - $10 \mathrm{wk}$ ) and old (26 weeks) from both strains. Several RAS components are overexpressed in the kidney of SHR rats [34]. This study shows that ACE activity increases in the intestine of otherwise healthy animals, but the increase was not more pronounced in animals which are genetically susceptible to hypertension and have an elevated expression of RAS components.

The ACE activity in the tissue lysates normalized to tissue protein levels was low but measurable using the Hip-His-Leu method. Hip-His-Leu is considered to be a specific substrate of ACE in various tissue lysates [35]. However, ACE activity was not completely inhibited by captopril in pilot in vitro experiments using intestine tissue lysates (data not shown). Therefore, it is possible that some of the measured HL formation could be due to other peptidase activities in the gut, a tissue which is specialized in digesting proteins and other nutrients. On the other hand, early studies on intestinal ACE showed that three peaks of ACE activity could be separated and purified by Sepharyl gel filtration from human intestinal homogenates [36] and all of them could be inhibited by captopril. Diet can influence ACE activity in the intestinal epithelium. A diet rich in proline (e.g. gelatin)

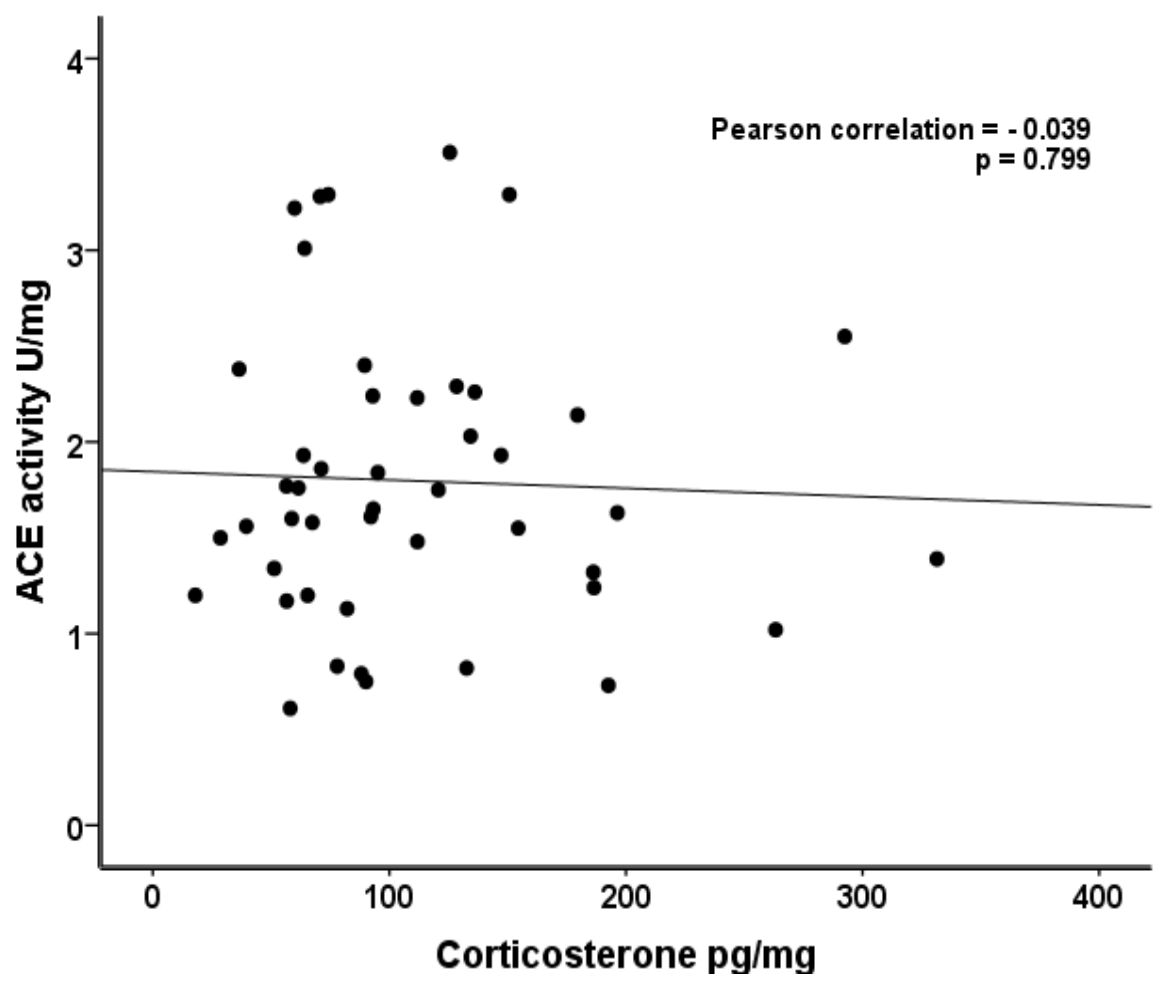

Figure 3. Correlation of ACE activity and corticosterone production in the gut $(n=46)$. 
particularly effectively increases intestinal levels of two prolyl peptidases namely ACE and dipeptidyl peptidase IV [37] [38]. This favours the proposal that at least part of the measured intestinal ACE activity may be digestive peptidase activity and this activity is inducible.

We found that young hypertensive SHRs had a higher activity of ACE in ileum compared to their normotensive WK counterparts, while in old rats, the situation was opposite. This is in disagreement with the findings of Costerousse et al. [39] that the membrane bound ACE activity as well as Ace mRNA amount were significantly higher in the normotensive rat strain than in their stroke-prone hypertensive relatives. These authors stressed that in addition to plasma ACE levels, genetic variability also determined differential Ace mRNA and membrane bound enzyme levels in somatic tissues. However, it is difficult to explain why this genetic determination would be opposite in young rats in the present study. It is not known whether the actual blood pressure levels in young SHRs vs malignant hypertension in stroke prone SHRs could provide some explanation.

In many post-mortem human tissues, ACE activity has been detected mainly in the membrane fraction of the homogenates [40]. It has long been known that the lung possesses high ACE activity and it has been considered as a reference tissue, although interestingly, kidney, duodenum and ileum, as well uterus have displayed higher activities than the lung. The authors suggested that ACE may possess a digestive or detoxifying role in the small intestine and kidney, as well as functioning to activate angiotensin I. The hypothesis that ACE could be viewed as a digestive enzyme for dietary peptides was postulated by Erickson et al. (1992) [4] and is supported by the discussion presented above.

In the elderly population, a chronic subclinical inflammatory status coexists with immune dysfunction. Special interest has been focussed on the intestine [41]. With aging of the intestinal epithelium, ischemic changes, low-grade inflammation and the increased use of nonsteroidal anti-inflammatory drugs hamper the normal function and regenerative capacity of the epithelial barrier which can lead to a mucosal injury [26] [42]. In addition, hypertension has been proposed to induce a low-grade inflammation in the gut epithelium [43]. We hypothesized that inflammatory changes could be balanced with increased corticosterone production, but no correlation was found between these two variables. In our previous studies, ACE release from the intestinal membranes was associated with increased corticosterone production following dextran sodium sulfate-induced colitis, a treatment which causes a more severe inflammation compared with the low-grade inflammation associated with age- and hypertension-induced changes [23]. Furthermore, we reported that angiotensin II increases intestinal corticosterone production in healthy intestine in vitro [23]. The interaction between the local RAS and corticosteroid synthesis in vasculature, heart and brain has been reviewed and discussed (MacKenzie et al., 2002) [44]. 
Age, as such, is an important factor which influences Ace gene expression, plasma and tissue levels of ACE activity. Costerousse et al. [45] studied these parameters in 1-day to 3-month old rats and detected a progressive and prominent increase of both Ace mRNA and the microsomal ACE concentration in the lung. The elevation in plasma levels was related to the maturation of thyroid function. Interestingly, intestinal Ace mRNA and ACE activity were very high at birth and then rapidly declined during the next two weeks. One can speculate that this is related with weaning from their dams and changing diets, similarly to the change occurring in epithelial lactase activity in the gut [46]. It would be enlightening to examine whether the differences were more marked in comparison to very old animals. Our findings and previous studies [47] stress that in addition to the strain of mice or rats, their age and gender are extremely important in planning and performing a physiological experiment.

\section{Conclusion}

We have compared ACE activity and corticosterone production in the small and large intestines of young and old SHR and WK rats. We reveal that ACE activity increases in the small intestine of both normotensive and hypertensive rats with age but in large intestine, the increase occurs only in the normotensive WK animals. We also show that the level of ACE activity is strain-dependent in the gut i.e. ACE activity is lower in the old SHR rats compared with their WK counterparts. Corticosterone production did not show any dependency on age or blood pressure, in support of the evidence that the inflammation associated with aging or hypertension is relatively mild. We suggest that low-grade inflammatory processes due to aging or hypertension or together are not strong enough stimuli to trigger a clear increase in corticosterone production, at least in in vitro conditions. On the other hand, the prominent increase in ACE activity in healthy animals points towards the presence of a low-grade inflammation in the gut epithelium related to aging. Further studies on markedly older normotensive and hypertensive animals will be needed to resolve the present dilemma.

\section{Acknowledgements}

We are sincerely grateful to Dr. Ewen McDonald for the language revision. Grants: H.S. Foundation for Clinical Chemistry, H.V. Finska Läkaresällskapet, Einar och Karin Stroem's Foundation, Finland.

\section{References}

[1] Gonzalez-Villalobos, R.A., et al. (2013) Rediscovering ACE: Novel Insights into the Many Roles of the Angiotensin-Converting Enzyme. Journal of Molecular Medicine (Berlin, Germany), 91, 1143-1154. https://doi.org/10.1007/s00109-013-1051-z

[2] Ward, P.E., et al. (1980) Angiotensin I Converting Enzyme (Kininase II) of the Brush Border of Human and Swine Intestine. Biochemical Pharmacology, 29, 1525-1529. https://doi.org/10.1016/0006-2952(80)90603-6

[3] Ward, P.E. and Sheridan, M.A. (1982) Angiotensin I Converting Enzyme of Rat In- 
testinal and Vascular Surface Membrane. Biochimica et Biophysica Acta, 716, 208-216. https://doi.org/10.1016/0304-4165(82)90270-7

[4] Erickson, R.H., et al. (1992) Rat Intestinal Angiotensin-Converting Enzyme: Purification, Properties, Expression, and Function. American Journal of Physiology, 263, G466-473.

[5] Shi, Y., et al. (2016) Activation of the Renin-Angiotensin System Promotes Colitis Development. Scientific Reports, 6, Article ID: 27552. https://doi.org/10.1038/srep27552

[6] Mizushima, T., et al. (2010) Blockage of Angiotensin II Type 1 Receptor Regulates TNF-Alpha-Induced MAdCAM-1 Expression via Inhibition of NF-KappaB Translocation to the Nucleus and Ameliorates Colitis. American Journal of Physiology-Gastrointestinal and Liver Physiology, 298, G255-266.

https://doi.org/10.1152/ajpgi.00264.2009

[7] Chappell, M.C. (2012) Nonclassical Renin-Angiotensin System and Renal Function. Comprehensive Physiology, 2, 2733-2752. https://doi.org/10.1002/cphy.c120002

[8] Liu, T.J., et al. (2016) AT1R Blocker Losartan Attenuates Intestinal Epithelial Cell Apoptosis in a Mouse Model of Crohn's Disease. Molecular Medicine Reports, 13, 1156-1162. https://doi.org/10.3892/mmr.2015.4686

[9] Inokuchi, Y., et al. (2005) Amelioration of 2,4,6-Trinitrobenzene Sulphonic Acid Induced Colitis In Angiotensinogen Gene Knockout Mice. Gut, 54, 349-356. https://doi.org/10.1136/gut.2003.036343

[10] Katada, K., et al. (2008) Dextran Sulfate Sodium-Induced Acute Colonic Inflammation in Angiotensin II Type 1a Receptor Deficient Mice. Inflammation Research, 57, 84-91. https://doi.org/10.1007/s00011-007-7098-y

[11] Jahovic, N., et al. (2005) The Effect of Angiotensin-Converting Enzyme Inhibitors on Experimental Colitis in Rats. Regulatory Peptides, 130, 67-74. https://doi.org/10.1016/j.regpep.2005.03.009

[12] Lee, C., et al. (2014) Enalapril Inhibits Nuclear Factor-KappaB Signaling in Intestinal Epithelial Cells and Peritoneal Macrophages and Attenuates Experimental Colitis in Mice. Life Sciences, 95, 29-39. https://doi.org/10.1016/j.lfs.2013.11.005

[13] Sueyoshi, R., et al. (2013) Angiotensin Converting Enzyme-Inhibitor Reduces Colitis Severity in an IL-10 Knockout Model. Digestive Diseases and Sciences, 58, 3165-3177. https://doi.org/10.1007/s10620-013-2825-4

[14] Koga, H., et al. (2008) Transanal Delivery of Angiotensin Converting Enzyme Inhibitor Prevents Colonic Fibrosis in a Mouse Colitis Model: Development of a Unique Mode of Treatment. Surgery, 144, 259-268. https://doi.org/10.1016/j.surg.2008.03.043

[15] Okawada, M., et al. (2011) Use of Enterally Delivered Angiotensin II Type Ia Receptor Antagonists to Reduce the Severity of Colitis. Digestive Diseases and Sciences, 56, 2553-2565. https://doi.org/10.1007/s10620-011-1651-9

[16] Spencer, A.U., et al. (2007) Reduced Severity of a Mouse Colitis Model with Angiotensin Converting Enzyme Inhibition. Digestive Diseases and Sciences, 52, 1060-1070. https://doi.org/10.1007/s10620-006-9124-2

[17] Santiago, O.I., et al. (2008) An Angiotensin II Receptor Antagonist Reduces Inflammatory Parameters in Two Models of Colitis. Regulatory Peptides, 146, 250-259. https://doi.org/10.1016/j.regpep.2007.10.004

[18] Salmenkari, H., et al. (2017) Orally Administered Angiotensin-Converting Enzyme-Inhibitors Captopril and Isoleucine-Proline-Proline Have Distinct Effects on Local Renin-Angiotensin System and Corticosterone Synthesis in Dextran Sulfate 
Sodium-Induced Colitis in Mice. Journal of Physiology and Pharmacology, 68, 355-362.

[19] Simoes e Silva, A.C., et al. (2013) ACE2, Angiotensin-(1-7) and Mas Receptor Axis in Inflammation and Fibrosis. British Journal of Pharmacology, 169, 477-492. https://doi.org/10.1111/bph.12159

[20] Taves, M.D., Gomez-Sanchez, C.E. and Soma, K.K. (2011) Extra-Adrenal Glucocorticoids and Mineralocorticoids: Evidence for Local Synthesis, Regulation, and Function. American Journal of Physiology-Endocrinology and Metabolism, 301, E11-E24. https://doi.org/10.1152/ajpendo.00100.2011

[21] Bouguen, G., et al. (2015) Intestinal Steroidogenesis. Steroids, 103, 64-71. https://doi.org/10.1016/j.steroids.2014.12.022

[22] Cima, I., et al. (2004) Intestinal Epithelial Cells Synthesize Glucocorticoids and Regulate T Cell Activation. The Journal of Experimental Medicine, 200, 1635-1646. https://doi.org/10.1084/jem.20031958

[23] Salmenkari, H., et al. (2015) Local Corticosterone Production and Angiotensin-I Converting Enzyme Shedding in a Mouse Model of Intestinal Inflammation. World Journal of Gastroenterology, 21, 10072-10079.

https://doi.org/10.3748/wjg.v21.i35.10072

[24] Mueller, M., et al. (2006) The Nuclear Receptor LRH-1 Critically Regulates Extra-Adrenal Glucocorticoid Synthesis in the Intestine. The Journal of Experimental Medicine, 203, 2057-2062. https://doi.org/10.1084/jem.20060357

[25] Kostadinova, F., et al. (2014) Why Does the Gut Synthesize Glucocorticoids? Annals of Medicine, 46, 490-497. https://doi.org/10.3109/07853890.2014.932920

[26] Meier, J. and Sturm, A. (2009) The Intestinal Epithelial Barrier: Does It Become Impaired with Age? Digestive Diseases, 27, 240-245.

https://doi.org/10.1159/000228556

[27] Soenen, S., et al. (2016) The Ageing Gastrointestinal Tract. Current Opinion in Clinical Nutrition \& Metabolic Care, 19, 12-18. https://doi.org/10.1097/MCO.0000000000000238

[28] Jeong, J.H., et al. (2017) Microvasculature Remodeling in the Mouse Lower Gut during Inflammaging. Scientific Reports, 7, Article No. 39848. https://doi.org/10.1038/srep39848

[29] Siltari, A., et al. (2017) Long-Term Feeding with Bioactive Tripeptides in Aged Hypertensive and Normotensive Rats: Special Focus on Blood Pressure and Bradykinin-Induced Vascular Reactivity. Journal of Physiology and Pharmacology, 68, 407-418.

[30] Oliveira, E.M., Santos, R.A. and Krieger, J.E. (2000) Standardization of a Fluorimetric Assay for the Determination of Tissue Angiotensin-Converting Enzyme Activity in Rats. Brazilian Journal of Medical and Biological Research, 33, 755-764. https://doi.org/10.1590/S0100-879X2000000700005

[31] Dinh, Q.N., et al. (2014) Roles of Inflammation, Oxidative Stress, and Vascular Dysfunction in Hypertension. BioMed Research International, 2014, Article ID: 406960.

[32] Virdis, A., Dell'Agnello, U. and Taddei, S. (2014) Impact of Inflammation on Vascular Disease in Hypertension. Maturitas, 78, 179-183. https://doi.org/10.1016/j.maturitas.2014.04.012

[33] Harvey, A., Montezano, A.C. and Touyz, R.M. (2015) Vascular Biology of Ageing-Implications in Hypertension. Journal of Molecular and Cellular Cardiology, 83, 112-121. https://doi.org/10.1016/j.yjmcc.2015.04.011 
[34] Williamson, C.R., et al. (2017) Comparative Analysis of Renin-Angiotensin System (RAS)-Related Gene Expression between Hypertensive and Normotensive Rats. Medical Science Monitor Basic Research, 23, 20-24. https://doi.org/10.12659/MSMBR.901964

[35] Kapiloff, M.S., et al. (1984) A Fluorometric Assay for Angiotensin-Converting Enzyme Activity. Analytical Biochemistry, 140, 293-302. https://doi.org/10.1016/0003-2697(84)90167-2

[36] Hayakari, M., et al. (1989) Purification of Angiotensin-Converting Enzyme from Human Intestine. Advances in Experimental Medicine and Biology, 247B, 365-370. https://doi.org/10.1007/978-1-4615-9546-5_60

[37] Suzuki, Y., et al. (1993) Dietary Regulation of Rat Intestinal Angiotensin-Converting Enzyme and Dipeptidyl Peptidase IV. American Journal of Physiology, 264, G1153-G1159. https://doi.org/10.1152/ajpgi.1993.264.6.G1153

[38] Yoshioka, M., Erickson, R.H. and Kim, Y.S. (1988) Digestion and Assimilation of Proline-Containing Peptides by Rat Intestinal Brush Border Membrane Carboxypeptidases. Role of the Combined Action of Angiotensin-Converting Enzyme and Carboxypeptidase P. The Journal of Clinical Investigation, 81, 1090-1095. https://doi.org/10.1172/JCI113421

[39] Costerousse, O., et al. (1999) Interstrain Differences in Angiotensin I-Converting Enzyme mRNA and Activity Levels. Comparison between Stroke-Prone Spontaneously Hypertensive Rats and Wistar-Kyoto Rats. Clinical and Experimental Hypertension, 21, 377-393. https://doi.org/10.3109/10641969909068671

[40] Lieberman, J. and Sastre, A. (1983) Angiotensin-Converting Enzyme Activity in Postmortem Human Tissues. Laboratory Investigation, 48, 711-717.

[41] Schiffrin, E.J., et al. (2010) The Inflammatory Status of the Elderly: The Intestinal Contribution. Mutation Research, 690, 50-56. https://doi.org/10.1016/j.mrfmmm.2009.07.011

[42] Sipos, F., Leiszter, K. and Tulassay, Z. (2011) Effect of Ageing on Colonic Mucosal Regeneration. World Journal of Gastroenterology, 17, 2981-2986. https://doi.org/10.3748/wjg.v17.i25.2981

[43] Santisteban, M.M., et al. (2017) Hypertension-Linked Pathophysiological Alterations in the Gut. Circulation Research, 120, 312-323. https://doi.org/10.1161/CIRCRESAHA.116.309006

[44] MacKenzie, S.M., et al. (2002) Local Renin-Angiotensin Systems and Their Interactions with Extra-Adrenal Corticosteroid Production. Journal of the Renin-Angiotensin-Aldosterone System, 3, 214-221. https://doi.org/10.3317/jraas.2002.043

[45] Costerousse, O., et al. (1994) Regulation of ACE Gene Expression and Plasma Levels during Rat Postnatal Development. American Journal of Physiology, 267, E745-E753. https://doi.org/10.1152/ajpendo.1994.267.5.E745

[46] Peuhkuri, K., et al. (1997) Age and Continuous Lactose Challenge Modify Lactase Protein Expression and Enzyme Activity in Gut Epithelium in the Rat. Journal of Physiology and Pharmacology, 48, 719-729.

[47] Quassinti, L., et al. (2007) Comparison of ACE Activity in Amphibian Tissues: Rana Esculenta and Xenopus laevis. Comparative Biochemistry and Physiology Part A: Molecular \& Integrative Physiology, 146, 119-123.

https://doi.org/10.1016/j.cbpa.2006.09.016 